Revista de Economia Política, vol. 34, nº 1 (134), pp. 158-180, janeiro-março/2014

\title{
Políticas productivas y competitividad industrial. El caso de Argentina y Brasil
}

\author{
MARTA BEKERMAN \\ GONZALO DALMASSO*
}

Productive policies and industrial competitiveness: The case of Argentina and Brazil.

This article aims, on the one hand, to analyze the increase of productive asymmetries between Argentina and Brazil that have been evidencing during the last two decades, and are currently reveled in the structural trade deficit of industrial products that affects Argentina in the bilateral relationship. On the other hand, it intends to contribute to understanding the roots of these asymmetries based on the differences in the public policies implemented by both countries during the period extending from the implementation of the Mercosur, in the early 1990s, until 2008. The focus is set on the technological pattern of industrial production and trade structures, considering a non neutral impact over the long term development.

Keywords: Argentina; Brazil; regional integration; productive asymmetries; industrial policy; economic development.

JEL Classification: O14.

\section{INTRODUCCIÓN}

La crisis europea nos está dejando un conjunto de lecciones que se vinculan, más allá del alto endeudamiento que muestran algunos países, con los problemas derivados de la falta de convergencia en materia de competitividad, lo que fue generando una profunda brecha entre las economías más fuertes y las más débiles. En todos los procesos de integración regional pueden existir mayores o menores diferencias iniciales entre los países que se integran. El desafío es, hasta qué punto la marcha de esos procesos contribuye a acercar los niveles de competitividad de esos países. Es

\footnotetext{
"Directora del Centro de Estudios de la Estructura Económica (CENES-UBA, Investigadora Principal del CONICT. E-mail: martabekerman@arnet.com.ar; Investigador del Centro CENES-UBA. E-mail: tuccid@hotmail.com. Submetido: 30/maio/2012; Aprovado: 9/janeiro/2013.
} 
que los mayores beneficios que pueden obtenerse de los procesos de integración son aquellos de carácter dinámico que permiten generar, en los países que se integran, cambios tecnológicos, efectos aprendizaje y de especialización productiva que permitan fortalecer la competitividad global de la región frente al resto del mundo.

¿Cuál es la realidad que existe hoy en el Mercosur y en particular en la relación entre Argentina y Brasil? Al momento de la firma del tratado de Asunción existían diferencias de tamaño de mercado y de niveles de industrialización entre ambos países. Pero esas diferencias lejos de haberse reducido se han visto profundizadas a lo largo de las últimas dos décadas. Esto se ve reflejado en las relaciones comerciales que muestran en los últimos años un superávit comercial creciente, favorable a Brasil, en el campo de las manufacturas industriales.

Es cierto que, a diferencia de Europa, nuestros países no posen una moneda única. Pero también es cierto que, en la medida en que las divergencias competitivas se hagan mas profundas será más difícil consolidar el proceso de integración.

En este trabajo nos proponemos analizar en forma comparativa la evolución productiva de ambas economías durante los últimos 20 años y tratar de determinar cuales fueron las principales políticas que influyeron en la profundización de las asimetrías productivas que están afectando en forma negativa al proceso de integración. Comprender donde están las raíces de ese proceso puede ser un punto de partida para poder avanzar hacia un proceso de armonización que permita brindar nuevos impulsos al Mercosur.

Nuestra hipótesis es que ciertas políticas públicas implementadas en Brasil y en la Argentina pueden haber contribuido a la profundización de esas asimetrías. Es que, si bien sus modelos de acumulación parecen haber sufrido la influencia de paradigmas muy similares, las políticas industriales implementadas por ambos países - ya sea por acción o por omisión - generaron impactos diferentes sobre la estructura productiva.

El trabajo analiza, en la primera sección, la evolución de la producción y de las exportaciones industriales de Brasil y Argentina desde principios de los años 1990 - cuando se firmó el Tratado de Asunción que dio origen al Mercosur - hasta la actualidad.

En la segunda sección hace eje en ciertas políticas públicas llevadas adelante por ambos países durante ese período, a nivel global y sectorial, que pueden ayudar a explicar el proceso de profundización de las asimetrías productivas que marca nuevos desafíos para el proceso de integración.

Por último se presentan las conclusiones del trabajo.

\section{PRODUCCIÓN INDUSTRIAL Y EXPORTACIONES DE ARGENTINA Y BRASIL}

\section{La evolución de la estructura industrial}

El análisis comparativo de la evolución de la producción física de la industria manufacturera durante las últimas dos décadas arroja dos resultados claros: 
1. La industria brasileña y la argentina crecieron a tasas muy similares a nivel consolidado; y

2. En el patrón sectorial de dicho crecimiento se observa en Brasil un peso significativamente mayor de los sectores de contenido tecnológico alto y medio-alto en relación a la Argentina. (Véase el Gráfico 1 y Cuadros 2 y 3 en el Anexo).

Entre los sectores que presentan mayor crecimiento relativo en Brasil con respecto a la Argentina se encuentran, entre otros, todas las ramas de bienes de capital, los equipos de informática y de comunicaciones, el complejo automotriz, la aeronáutica (en "otros equipos de transporte"). Mientras que, entre en los sectores en los que la industria argentina muestra una mejor perfomance relativa se encuentran aquéllos de menor contenido tecnológico: alimentos y bebidas, productos de madera, caucho y plástico, cueros y calzado, metales comunes y productos minerales no metálicos. Cabe destacar, no obstante, que si bien estos sectores ya tienen un grado de madurez tecnológica que les da un bajo grado de dinamismo innovativo a nivel global, la Argentina ha logrado converger en algunos de ellos (petroquímica, siderurgia, aluminio, alimentos y madera) a la frontera tecnológica global (ver Bernat \& Corso, 2010). De hecho, algunas empresas nacionales han contrubuido a expandirla, como es el caso de los tubos sin costura de Techint. Esta asimetría en el patrón sectorial de crecimiento industrial puede observarse en el Gráfico $1{ }^{1}$

El Cuadro 2 en el Anexo muestra las diferencias de crecimiento que muestran los distintos sectores a lo largo de tres subperíodos: 1993-1998; 1998-2003 y 2003-2008. Durante los años 1990, la Argentina tuvo un mayor crecimiento relativo de los sectores intensivos en recursos naturales (alimentos, comodities industriales, destacándoselos metales comunes y los plásticos), mientras que en Brasil, se destaca la evolución de las maquinarias y equipos eléctricos, explicada en gran medida por el shock de demanda de las empresas privatizadas, como veremos posteriormente. Por su parte, tanto las máquinas para escritorio y los equipos de informática, como los relojes e instrumentos médicos y las maquinarias y equipos mecánicos ampliaron la brecha en relación a la Argentina, que muestra un desempeño muy pobre frente a un crecimiento moderado en Brasil.

Es durante el segundo período, el de la crisis de fines de los años 1990, cuando se profundiza la brecha cualitativa entre ambas estructuras productivas. En efecto, dicha crisis se convirtió en una depresión sistémica en la industria argentina, con efectos más marcados sobre los sectores tecnológicamente más intensivos: las maquinarias eléctricas, los electrónicos y los automotores y los otros equipos de trans-

\footnotetext{
${ }^{1}$ Se debió recurrir a índices de volumen físico de producción por una cuestión de disponibilidad de datos. Por lo tanto hay que mirar con cierto reparo las tasas de crecimiento sectoriales ya que estos índices no reflejan la sustitución de insumos locales por importados que se ha dado desde los 1990. Esto ha reducido el coeficiente de VAB/VBP y puede haber afectado, particularmente a las industrias tecnológicamente más complejas. No obstante, a nivel relativo la conclusión sigue siendo válida, dado que dicho fenómeno se ha producido en ambos países.
} 
Gráfico 1: Argentina y Brasil. Tasas comparadas de crecimiento industrial por sectores - 1993-2008 Variaciones promedio anual acumulado de producción física (\%)

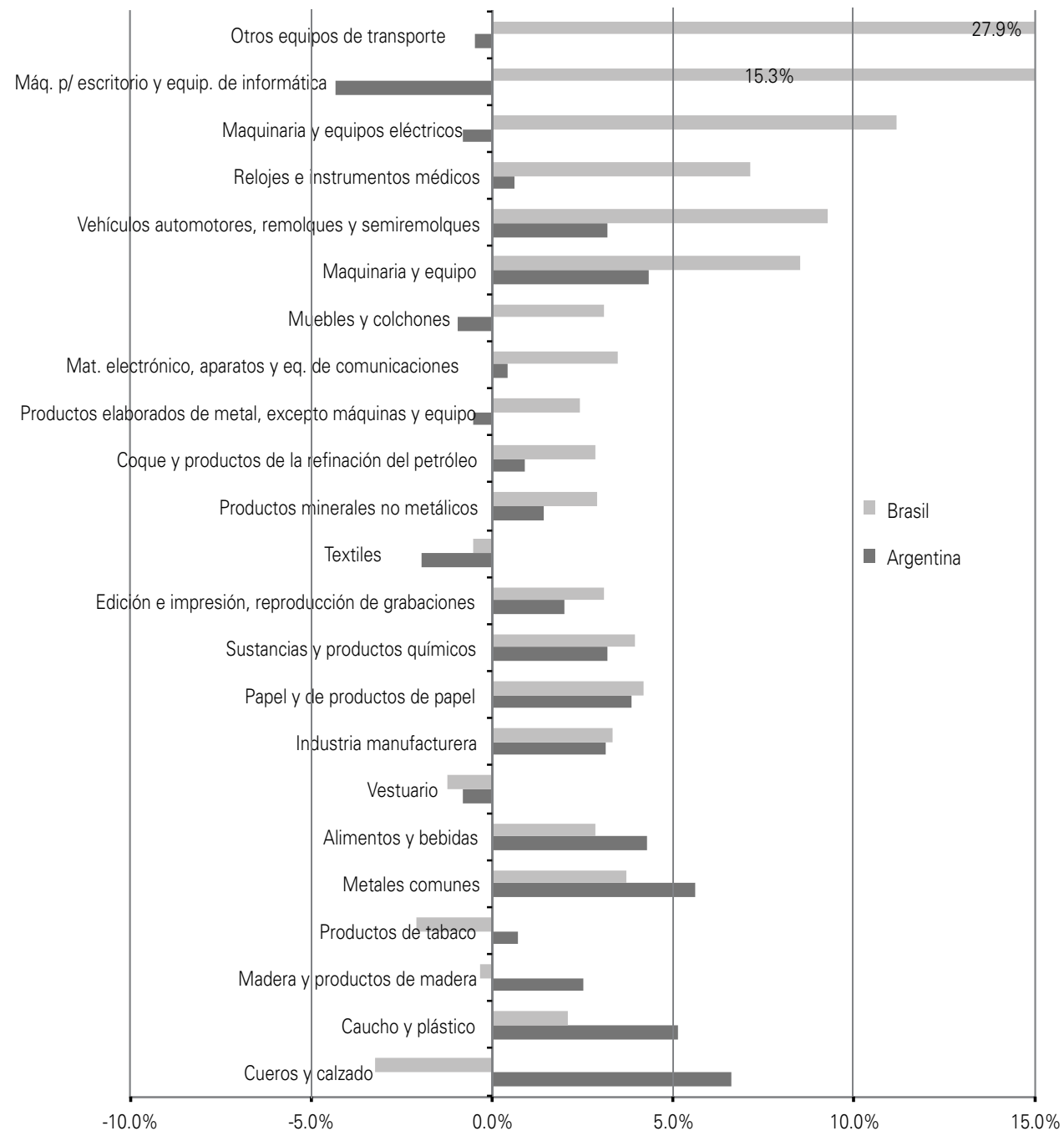

Fuente: Elaboración propia en base a IGBE, INDEC y Centro de Estudios de la Producción (CEP).

porte presentan caídas superiores al 9\% anual entre 1998 y 2003. En Brasil, en contraste, la devaluación temprana logró evitar que la crisis se profundizara y su PBI no cayó en ningún año del período. Además la reversión de los precios relativos permitió un despegue de los sectores más dinámicos desde el punto de vista tecnológico, destacándose los "otros equipos de transporte" (i.e., cadena aeronáutica) que expandió fuertemente sus exportaciones, como veremos en el punto siguiente.

En el caso del complejo automotriz, el sector cae un $9 \%$ en la Argentina contra un crecimiento del $4 \%$ en Brasil. Se produjo, entonces, una importante relocaliza- 
ción de automotrices multinacionales desde el primero hacia el segundo, las que fueron acompañadas por muchas empresas autopartistas. Por otro lado algunos segmentos de maquinarias y equipos de producción quedaron casi desmantelados en la Argentina durante este período, como es el caso de las máquinas-herramientas, (cayeron desde más de 4 mil toneladas en 1990 a sólo 306 en el año 2002). ${ }^{2}$

La recuperación de la industria argentina luego de la devaluación de 2002 fue tan espectacular como la crisis previa. Con altas cifras de crecimiento, superiores al 9\% medio anual y alcanzando a todas las ramas, revirtió una tendencia de más de 30 años hacia la pérdida de participación en el PBI. Los sectores de mayor dinamismo fueron los que habían sido más afectados durante los 1990 y la crisis posterior, destacándose los bienes de capital: maquinaria y equipos, materiales y equipos eléctricos, electrónicos, instrumentos médicos y el complejo automotriz, incluyendo la maquinaria agrícola. Además, crecieron a altas tasas los alimentos y bebidas, las industrias tradicionales mano de obra-intensivas ligadas al mercado interno (madera y muebles, textil y confecciones, y cuero y calzado) y los commodities industriales, como la metalurgia, y los minerales no metálicos - dentro de los que se hallan los materiales de contrucción, que respondieron al fuerte shock de demanda por el boom de este sector.

Si comparamos en términos cuantitativos el desempeño de ambas economías durante el período 2002-2008, vemos que el crecimiento de la industria argentina más que duplicó al de la brasileña. Debemos tomar en cuenta que Argentina venía de una profunda depresión -en contraste con lo acontecido en Brasil- por lo que arrancó la reactivación desde niveles de producción muy bajos y con una alta capacidad ociosa que implicarían un contenido significtivo de "efecto rebote" en las tasas de crecimiento del período.

Pero si bien el crecimiento agregado de la industria brasileña fue moderado, afectado por la política macroeconómica de inflation targeting, los sectores que mostraron un mayor crecimiento relativo son los tecnológicamente más dinámicos (los primeros seis sectores del Cuadro 2, anexo), y definidos como focos estratégico de la política industrial (ver segunda sección). De manera que, pese a la fuerte recuperación de la industria argentina, Brasil mantuvo la brecha en relación al crecimiento de los sectores tecnológicamente más complejos, y la amplió en algunos casos como el de los otros equipos de transporte y la maquinaria de oficina y equipos de informática, que recibieron fuerte estímulos desde las políticas públicas.

Las asimetrías señaladas han tendido a reflejarse en la pauta de exportaciones de cada país y en las condiciones del comercio bilateral, como veremos a continuación.

\section{La evolución de las exportaciones y del comercio bilateral}

Las últimas dos décadas de transformaciones de las economías de Brasil y Argentina han estado caracterizadas por su apertura al mercado internacional. Las

\footnotetext{
${ }^{2}$ Ver Estadísticas de Productos Industriales-INDEC.
} 
exportaciones de ambos países crecieron a una tasa media anual acumulada del $10 \%$ entre 1990 y 2003 - a un ritmo significativamente menor que las importaciones -, para acelerarse al $34 \%$ para Brasil y al $27 \%$ para la Argentina durante el período 2003-2008. En este contexto la participación de las exportaciones industriales sobre el total de bienes se han mantenido sin grandes alteraciones durante todo el período, representando una proporción significativamente más alta en el caso brasileño (ver Gráfico 2) ${ }^{3}$

Gráfico 2: Argentina y Brasil - Evolución de las exportaciones industriales (índice base 1990=1) Participación sobre las exportaciones totales de bienes (\%)

En U\$S FOB corrientes. Período 1989-2008

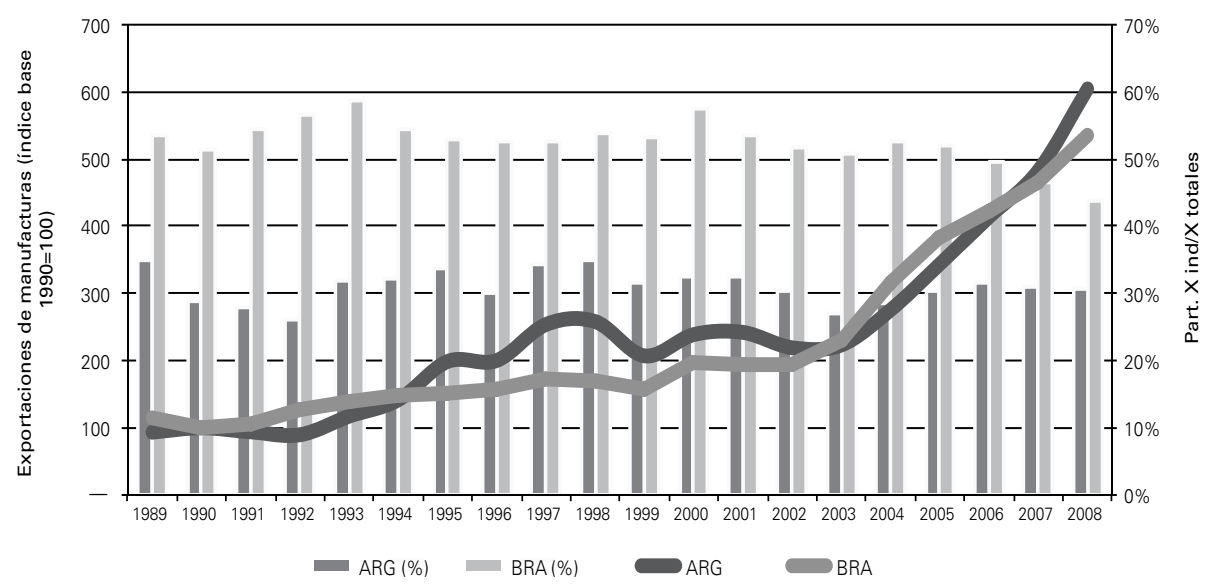

Fuente: Elaboración propia en base OMC.

Si observamos ahora específicamente el comportamiento de las exportaciones industriales según su contenido tecnológico - de acuerdo a la clasificación de la OCDE - se observan algunas similitudes y contrastes en la evolución de ambos países (ver Cuadro 3, del Anexo). Entre las similitudes está la fuerte caída de las industrias tradicionales (madera y muebles, textiles y calzados) y el aumento de participación de los combustibles, explicado en gran parte por la evolución de los precios. Además, ha ganado participación en ambos países el complejo automotriz, al amparo de los regímenes de promoción y del comercio regional intra-firma por parte de las multinacionales.

Pero el principal contraste se encuentra en la evolución de los sectores de alto contenido tecnológico, que han más que duplicado su participación en Brasil y se

\footnotetext{
${ }^{3}$ Desde 2006 se observa una tendencia descendente en Brasil dado el crecimiento exponencial de las exportaciones primarias tanto en precios como en cantidades. La Argentina, en cambio, aplicó una política de retenciones a exportaciones primarias que le permitió mantener la participación de sus exportaciones industriales.
} 
han mantenido en niveles muy bajos en Argentina. Esto es explicado por las exportaciones aeronáuticas (i.e., de la Empresa Brasileira Aeronautica - Embraer) y, en menor medida, por la evolución de los farmacéuticos y los equipos electrónicos y de comunicaciones. El Cuadro 3 del Anexo muestra una diferencia de ocho puntos en el peso de los sectores de alta y media-alta tecnología en Brasil con respecto a la Argentina (cinco puntos explicados por los de más alto contenido tecnológico).

Dentro de este último grupo, cobra particular relevancia la evolución de las exportaciones dentro del Mercosur (ver Cuadro 1). Desde su creación en los 1990, los flujos del comercio bilateral han mostrado un dinamismo muy superior a la corriente extra-bloque, y su composición ha quedado ampliamente dominada por manufacturas de origen industrial (MOI), a diferencia del comportamiento de las exportaciones al resto del mundo. En este sentido, el comercio regional ha adquirido un interés fundamental para Brasil como plataforma para la adquisición de economías de escala y el aprendizaje en materia de exportación de bienes tecnológicamente más complejos, para luego lanzarse con mayor competitividad hacia terceros mercados. Un claro ejemplo de este proceso lo encontramos en el caso de la telefonía celular (ver Bekerman \& Montagu, 2007).

Las asimetrías que se han generado en los últimos 20 años en la evolución de los sectores señalados han tenido su impacto en el intercambio bilateral, donde se ha consolidado un déficit estructural en MOI. Es que la Argentina fue perdiendo participación en el mercado de Brasil, mientras que sus importaciones desde este país no sólo muestran un fuerte crecimiento, sino también un claro desvío desde terceros mercados (Bekerman \& Montagu, 2007).

Cuadro 1: Argentina: Intercambio comercial con Brasil en bienes de contenido tecnológico alto y medio-alto (excl. transporte automotor) - en U\$S corrientes

\begin{tabular}{|l|c|c|c|}
\hline & $1994-1996$ & $2006-2008$ & Var. \% \\
\hline Exportaciones (FOB) & 658.037 .428 & 1.674 .553 .103 & $154 \%$ \\
\hline Importaciones (CIF) & 1.470 .253 .780 & 4.936 .792 .973 & $236 \%$ \\
\hline Balanza comercial & -812.216 .352 & -3.262 .239 .870 & $302 \%$ \\
\hline Corriente de comercio & 2.128 .291 .209 & 6.611 .346 .076 & $211 \%$ \\
\hline Déficit como \% del comercio & $38,2 \%$ & $49,3 \%$ & $+11,2 \mathrm{pp}$ \\
\hline Déficit como \% del comercio (incl. automotrz) & $18,8 \%$ & $32,6 \%$ & $+13,8 \mathrm{pp}$ \\
\hline
\end{tabular}

Fuente: Elaboración propia en base a Comtrade.

En particular, en el caso del sector de bienes de capital Brasil ha aumentado su penetración en el mercado argentino desde su recuperación y ha devenido su principal proveedor superando a los Estados Unidos. ${ }^{4}$ Esto generó que el desequilibrio comercial del sector aumentara del $50 \%$ a mediados de los 1990 a más del $70 \%$ de la corriente de comercio en 2006-2008 (véase Gráfico 3).

\footnotetext{
${ }^{4}$ Pero desde 2005 Brasil viene reduciendo su participación a manos de las maquinarias de origen chino.
} 
Gráfico 3: Intercambio bilateral de bienes de capital (excepto equipos de transporte) de Argentina con Brasil. Participación del rubro en los flujos de comercio de cada país.

Períodos 1995-1998 y 2003-2008 - en millones de U\$S corrientes yen porcentajes
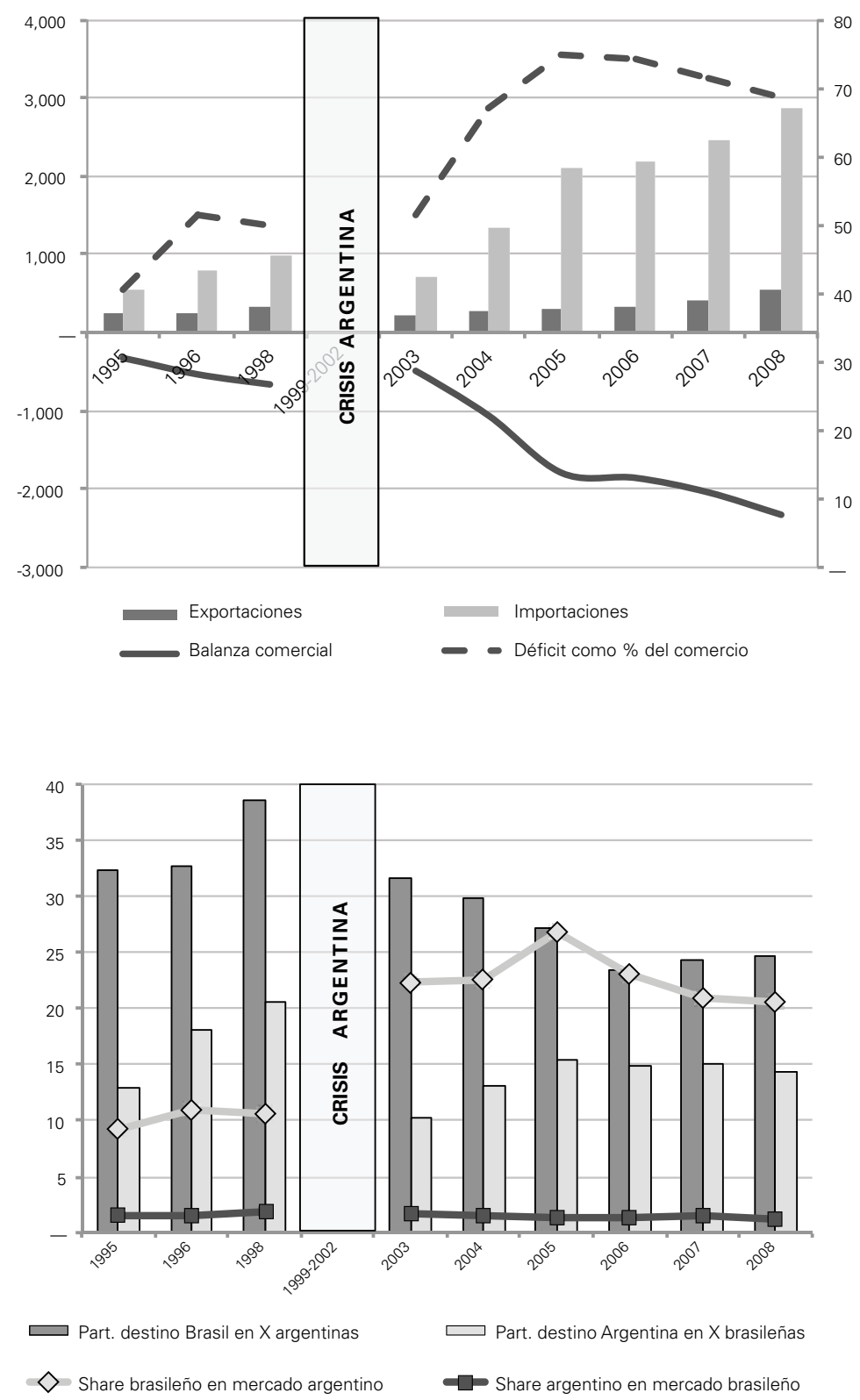

Fuente: Elaboración propia en base a Comtrade. 


\section{ESTRATEGIAS PRODUCTIVAS EN LOS ÚLTIMOS 20 AÑOS}

A partir de las asimetrías que hemos observado en la evolución de los patrones productivos y de exportación de Argentina y Brasil, en esta sección pasaremos a analizar ciertas diferencias más relevantes en las políticas productivas que se llevaron a cabo durante las últimas dos décadas.

\section{Las políticas de promoción industrial y la selectividad estratégica ${ }^{5}$}

Como veremos en las secciones posteriores, la apertura comercial que tuvo lugar en los años 1990 en Brasil tuvo un carácter relativamente gradual y discriminatorio, brindando una mayor protección efectiva a sectores de contenido tecnológico alto o medio-alto. Al mismo tiempo, las privatizaciones y la apertura a los capitales extranjeros tuvieron entre sus objetivos el desarrollo de proveedores locales y el derrame de externalidades de conocimiento y productividad, mientras que los instrumentos de promoción financiera tendieron a discriminar a favor de la producción nacional.

Como consecuencia de estas medidas, si bien no pudo evitar cierta desarticulación y pérdida de valor agregado en sus cadenas productivas, Brasil logró atenuar los impactos negativos de la apertura sobre la industria en general y sobre algunos sectores estratégicos en particular, y no vivió un proceso de desindustrialización con quiebras masivas de empresas como el que se dio en la Argentina (ver Bekerman \& Dalmasso, 2010).

En la Argentina de los 1990, en contraste, prácticamente todos los regímenes sectoriales fueron desmantelados y se restringió la política industrial a regímenes de carácter horizontal, como el bienes de capital y el Régimen de Especialización Industrial (REI) -que buscó fomentar la reconversión de la industria a través de su especialización y el incentivo a las exportaciones. La eficacia de este último fue muy limitada, dada la insuficiencia de los incentivos ofrecidos, la falta de conexión con políticas crediticias y tecnológicas adecuadas, y la no exigencia de compromisos concretos de reconversión productiva (Sirlin, 1999).

La gran excepción fue el régimen de promoción automotriz, que se ha mantenido desde 1991 hasta la actualidad. Este régimen -muy similar al que se implementó posteriormente en Brasil-, derivó en un régimen común en el ámbito del Mercosur y en un esquema de comercio bilateral administrado. Por otro lado, los regímenes sectoriales ad hoc implícitos en las medidas de protección comercial impuestas en algunos sectores amenazados por la competencia externa (ej. textiles) no contaron con ningún tipo de normativa que asegurara o incentivara un proceso

\footnotetext{
${ }^{5}$ Esta sección está basada en dos Documentos de Trabajo publicados anteriormente por el CENES, que realizan un análisis comparativo detallado de las políticas industriales en Argentina y Brasil: Bekerman, et al., 1995, y Bekerman y Dalmasso, 2010.
} 
de reconversión productiva que los pudiera tornar internacionalmente competitivos (véase Bekerman et al., 1995).

Durante la década de los 2000, si bien ambos países han compartido una mayor convicción sobre la necesidad de intervenciones activas sobre el desarrollo productivo, se observa una diferencia en la orientación de la política económica: Brasil ha colocado mayor énfasis en aspectos microeconómicos, orientados a la competitividad de su industria y de sus sectores estratégicos, como puede verse en los programas implementados durante la década; mientras tanto, la Argentina basó más su política de competitividad en los aspectos macroeconómicos, a través de políticas de expansión de la demanda y del sostenimiento de un tipo de cambio real estable y competitivo.

Las políticas selectivas fueron inicialmente excepcionales en la Argentina, aunque en los últimos años se observa la aparición de nuevos incentivos fiscales y líneas de crédito para sectores específicos, como bienes de capital, software, maquinaria agrícola, motos y moto-partes y biocombustibles.

En Brasil, por su parte, se pusieron en marcha programas de desarrollo productivo de carácter integral y articulados con programas de infraestructura, salud y educación. ${ }^{6}$ Abarcaron objetivos de modernización industrial, expansión exportadora e incentivo a la innovación, combinando medidas de promoción sistémicas con otras destinadas a sectores específicos. La Política Industrial, Tecnológica y de Comercio Exterior de Brasil (PITCE) implementada en 2003 se orientó hacia estos objetivos e hizo posible que Brasil recuperara cierta capacidad de formular y coordinar políticas industriales a través de distintos instrumentos necesarios para estimular la competitividad (Ferraz et al., 2010).

Su política macroeconómica, en tanto, tuvo una estrategia de inflation targeting, que se manifestó en altas tasas de interés y un tipo de cambio real que tendió fuertemente a la baja. Ello le permitió mantener una baja tasa de inflación y una significativa afluencia de capitales, pero tuvo efectos negativos sobre la tasa de inversión y la competitividad-precio de la industria restringiendo así los efectos de los planes de desarrollo.

Pero más allá de estas diferencias, un rasgo común a ambas estrategias durante los últimos años ha sido la promoción - con perspectivas a largo plazo - de ciertas áreas intensivas en conocimiento e inductoras de cambio tecnológico a nivel transversal. Éstas son el sector de software y servicios IT, la biotecnología y la nanotecnología.. En el caso de Brasil, también se ha fortalecido el apoyo a la industria de bienes de capital e incluso se ha promovido la creación del sector de semi-conductores a través de la generación de clusters productivos. Si bien la Argentina está apoyando el desarrollo de la industria electrónica de consumo - a través del Régimen de Promoción de Tierra del Fuego - la producción nacional de componentes electrónicos no se encuentra entre sus prioridades.

Como corolario de nuestro período de análisis en su conjunto, podría señalar-

\footnotetext{
${ }^{6}$ Para un análisis más detallado de estos programas, véase Bekerman y Dalmasso (2010).
} 
se entonces que la selección y promoción de sectores estratégicos presenta en Brasil una mayor coherencia y constancia que en la Argentina. A nivel de sectores particulares, la privatización de las empresas núcleo de la industria aeronáutica ha tenido resultados muy diferentes en cada país. La privatización de la empresa Embraer, junto con el apoyo del BNDES, permitieron sanear con nuevas inversiones a la empresa núcleo del entramado productivo del sector y aumentar fuertemente su productividad. En la Argentina, en cambio, la privatización de la Fábrica Militar de Aviones - inaugurada en Córdoba en 1927 y pionera en América Latina en la producción de aviones a reacción - significó el desmantelamiento de la industria aeronáutica nacional.

Finalmente, un último punto importante destacar son las asimetrías en materia de capacidad institucional en lo que concierne a la política industrial. En las políticas de promoción (y especialmente en aquéllas de alcance sectorial), la constancia y la eficacia de los mecanismos de implementación suele ser tan importante como su diseño mismo. Y, en este sentido, veremos en la Argentina una proliferación de incentivos que se realizan desde diversos ámbitos y organismos estatales sin mostrar signos de coordinación. Coexisten en ese esquema nuevos programas que no prosperan y la duplicación de objetivos y de poblaciones beneficiarias. Podría señalarse que la ausencia de una política rectora de prioridades puede generar una importante pérdida de eficiencia en la asignación de los recursos (Bekerman \& Dalmasso, 2010).

En el caso de Brasil, la política de incentivos transitó años de perfeccionamiento con la implementación de diferentes mecanismos, que respondieron a una definición de metas estratégicas explícitas. Esto no quiere decir que Brasil haya resuelto sus fallas de articulación de políticas. En efecto, la PITCE ha mostrado ciertos problemas de coordinación entre sus instrumentos (ver Suzigan \& Furtado, 2006). Algunos de esas debilidades buscaron ser atendidas en el diseño de la segunda etapa del plan, implementado a partir de 2008, y denominado Política de Desarrollo Productivo (PDP). A través del mismo se realizaron cambios en su ingeniería institucional a fin optimizar la efectividad de los instrumentos y de los recursos asignados. Se diseñaron sistemas de coordinación y control mediante la conformación de un Consejo Gestor del programa y un sistema de feedback con el sector privado a través de los foros sectoriales de competitividad. De manera que la Política de Desarrollo Productivo de Brasil es considerada por algunos autores como el esfuerzo más avanzado de América Latina en términos de diseño y articulación de política industrial (Peres \& Primi, 2009).

\section{La política comercial}

En el campo del comercio exterior, Brasil ha mostrado tener una estrategia de inserción internacional más definida que la Argentina durante todo el período analizado, lo que se refleja particularmente en la coherencia y la constancia de los criterios de selectividad sectorial de su política comercial y en los instrumentos de promoción utilizados. Podemos observar que el conjunto de instrumentos de fo- 
mento a las exportaciones aparece como el más sólidamente implementado en Brasil. La combinación de desgravaciones impositivas aplicadas a la producción para la exportación con un financiamiento compatible en cantidad y costo con los estándares internacionales, ha permitido un aumento sostenido de las exportaciones brasileñas, aún en momentos en que se presentaron restricciones de acceso al financiamiento externo (Baruj et al., 2005). En Argentina, en contraste, los efectos combinados de la volatilidad del contexto [macroeconómico y cambiario] y las debilidades institucionales del sector público se han mostrado con especial intensidad en el campo de la promoción de exportaciones, cuya efectividad -a pesar de la diversidad de instrumentos- ha sido muy baja (Bouzas \& Cabello, 2007).

En primer término, es importante destacar las diferencias, ya señaladas, que se observaron en los procesos de apertura de los años 1990 en cada país, especialmente en lo que se refiere a su profundidad, el timing de su implementación y su selectividad.

El proceso de liberalización comercial en Brasil fue implementado gradualmente y evidenció una fuerte discriminación inter-sectorial. Los sectores privilegiados en este sentido fueron principalmente el complejo automotriz, la informática y los productos electrónicos (electrodomésticos, comunicaciones, y en especial la telefonía celular), textiles y calzados, ciertas ramas de los bienes de capital, la industria naval y la aeronáutica.

En contraposición, la apertura en la Argentina se dio en forma indiscriminada y con características de shock, con un tipo de cambio real mucho más sobrevaluado que el brasileño durante los primeros años de la reforma. El arancel nominal promedio llegó al 12\% en 1991 y sólo un pequeño grupo de productos (los automóviles, los textiles y el calzado) quedó sujeto a algún tratamiento especial. Los bloques de metalmecánica (especialmente maquinarias y equipos) y los electrónicos fueron los que mostraron menores tasas de protección efectiva, significativamente más bajas que las permitidas en el arancel externo común del Mercosur (Bouzas \& Cabello, 2007).

En síntesis, mientras que la Argentina se abrió más intensamente a los bienes de capital, TICs y electrónicos, buscando aumentar la productividad de su economía a partir de la incorporación de tecnologías externas, Brasil mantuvo un mayor grado de protección a su producción local en dichos sectores.

Más recientemente, durante los años 2000, Brasil ha perseguido una agresiva estrategia de expansión en el comercio mundial, dejando de lado el foco en la balanza comercial y haciendo eje en la promoción de exportaciones (Suzigan \& Furtado, 2006). Su objetivo es ampliar su base exportadora priorizando las actividades más intensivas en conocimiento. En este sentido, el BNDES y el Banco de Brasil otorgan líneas especiales de pre y post-financiación para los sectores estratégicos (mencionados en el punto anterior). En contraste, el financiamiento público a las exportaciones en la Argentina ha sido más limitado y no existieron durante el período directrices sectoriales marcadas en las líneas de crédito del Banco de la Nación Argentina (BNA) y del Banco de Inversión y Comercio Exterior (BICE), lo que analizaremos en el punto siguiente. 
Cabe destacar, por último, que el BNDES cuenta con un programa de apoyo a la internacionalización de empresas brasileras, condicionado a que sean proyectos que contribuyan a incrementar las exportaciones desde ese país. Fue así como corporaciones brasileñas han adquirido empresas argentinas líderes en el mercado local luego de la devaluación de 2002, en lo que se ha denominado como el fenómeno de las "translatinas" (véase en Bezchinsky et al., 2007).

Los mayores contrastes en las herramientas de promoción comercial, insistimos, se encuentran en los instrumentos financieros. Los incentivos tributarios utilizados han sido bastante similares en este período y se han orientado a remediar el sesgo anti-exportador de la protección arancelaria -fundamentalmente a través del mecanismo de drawback- y de evitar "exportar impuestos" a partir de la desgravación del IVA incidente sobre los bienes exportados. Se observa, sin embargo, una diferencia importante: en Brasil las modalidades de estos regímenes son la exención o suspensión impositiva, mientras que en la Argentina se otorga un reintegro como crédito fiscal sobre exportaciones ya concretadas. Ello implica una diferencia clave de competitividad en materia de financiamiento, especialmente si se tiene en cuenta que el mecanismo de pago del drawback ha sido históricamente muy lento, razón por la cual el régimen de admisión temporaria suele ser el más utilizado, aún cuando contiene una restricción en los plazos para realizar las operaciones (Bouzas \& Cabello, 2007).

\section{El financiamiento del desarrollo productivo}

Algunos estudios revelan que los sistemas financieros de los países del Mercosur muestran aún niveles de profundidad bajos o con un desarrollo menor que el que cabría esperar en función del PBI per cápita de la región. Pero surgen aquí diferencias muy marcadas dado que el nivel de profundidad de Brasil es muy superior al de la Argentina. Sin embargo no se observa en ambos casos la generación de una oferta de crédito suficiente para el sector privado, dado que los papeles negociados son en su mayoría de corto plazo (véase Fanelli, 2008).

La volatilidad de las variables macroeconómicas tiene profundas injerencias sobre el sistema financiero, al alterar el comportamiento microeconómico de los agentes generando un sesgo hacia los depósitos de corto plazo y a la dolarización del ahorro (Fanelli \& Frenkel, 1994), En la Argentina, particularmente, el problema de la volatilidad del producto ha persistido luego de la estabilización de los '90, en mucha mayor medida que en Brasil Y con ella, también ha subsistido la desconfianza en el sistema financiero local y la tendencia a fugar capitales durante las crisis y reingresarlos al país en inversiones físicas durante la reactivación. Es así como la inversión durante el período de recuperación que se inició en 2003 ha sido fundamentalmente autofinanciada. ${ }^{7}$ Esto tiene repercusiones sobre el patrón pro-

\footnotetext{
${ }^{7}$ El crédito como porcentaje del PBI se mantuvo cercano al 12\% en Argentina entre 2003 y 2008 (39\% en Brasil; más del 100\% en los países desarrollados), mientras que sólo el 6,8\% de las empresas privadas
} 
ductivo, ya que dicho tipo de financiamiento tiende a limitar el desarrollo de proyectos de mayor escala y con plazos de retorno más prolongados, imponiendo una restricción a las transformaciones estructurales.

En ese contexto, es en el rol de la política pública de financiamiento del desarrollo productivo, donde se observa una asimetría sustancial entre Brasil y la Argentina durante el período analizado.

Desde principios de los 1990, Brasil ha intensificado la participación de su banca pública - y del Banco Nacional de Desarrollo Económico y Social (BNDES) en particular - en el sistema financiero. El BNDES jugó un rol fundamental en el apoyo a las inversiones de las empresas privatizadas y sus proveedores locales en los 1990, en la reestructuración y expansión de empresas como Embraer y Petrobras, en el financiamiento del boom exportador de los 2000, de los sectores estratégicos seleccionados por los planes industriales, de proyectos innovadores y de los mega-planes de infraestructura de los últimos años. Mientras tanto, la Argentina cerró las puertas de su Banco Nacional de Desarrollo (BANADE) en 1992. En su lugar fue creado el Banco de Inversión y Comercio Exterior (BICE), un banco de segundo grado que es lo más asimilable al BNDES en cuanto a objetivos que existe actualmente en el país, pero cuyas dimensiones y alcance son incomparables, pese a la expansión de sus desembolsos durante la última década.

En este aspecto, cabe destacar que la asimetría en la política de financiamiento público al sector productivo que se observa en el período analizado está vinculada, a su vez, con la asimetría en el volumen de la banca pública (y del sistema financiero en general) en Brasil con respecto al de Argentina. Esto le permite al primero convertir a sus bancos estatales en instrumentos de promoción gravitantes en las inversiones, posibilidad que se ve muy limitada en el segundo.

Focalizándonos más concretamente en el accionar del BNDES durante nuestro período de análisis, se observa en primer término que este banco tuvo un rol central en el financiamiento de la reestructuración y la modernización de la industria durante el proceso de reformas de los 1990. Si bien cambió su eje hacia el financiamiento de la inversión de las empresas privatizadas -especialmente en infraestructura de energía y telecomunicaciones - y a las terminales automotrices multinacionales, dicho financiamiento otorgó privilegio a los proveedores nacionales, por lo que significó un fuerte incentivo a la demanda local de bienes intermedios y de capital, particularmente en los sectores de equipos de telecomunicaciones, equipos eléctricos y autopartes. También brindó líneas de financiamiento de largo plazo para la adquisición de software y servicios IT y a la demanda de equipos de telefonía celular - ambos desarrollados dentro de las fronteras de Brasil - y prestó un apoyo decisivo para la recuperación económica y competitiva de la EMBRAER.

Durante el período más reciente, el BNDES ha tenido un rol protagónico en la elaboración y gestión financiera de los programas de desarrollo productivo de

argentinas financiaron su inversión a través de crédito bancario (38\% en Brasil; sin datos para países desarrollados). Fuente: Banco Mundial. 
Brasil. El banco cuenta con líneas de apoyo a la inversión, producción, comercialización y exportación de bienes y servicios, priorizando las actividades más intensivas en conocimiento. Cuenta con al menos una línea de financiamiento preferencial para cada uno de los sectores estratégicos definidos en la política industrial. ${ }^{8}$ En el caso particular del sector de bienes de capital, sus líneas de pre-financiación de las exportaciones (más de dos años de plazo a tasa Libor) implican una reducción de su precio de venta en el mercado argentino del $20 \%$ en relación a los productores locales, debido al apalancamiento que representa para el fabricante (ADIMRA, 2010). A modo general, se observa que desde mediados de los 1990 hasta la fecha, los sectores hacia donde se han dirigido más intensamente los créditos del banco son los de contenido tecnológico alto y medio-alto, particularmente los otros equipos de transporte (aeronáutica), el complejo automotriz y las máquinas y equipamientos. Además, posee líneas especiales de financiamiento a actividades de I\&D y proyectos innovadores, algo que en la Argentina se encuentra en gran medida circunscripto al ámbito del Ministerio de Ciencia y Tecnología.

En la Argentina, en cambio, el financiamiento del sector productivo por parte de los bancos públicos federales ha sido más limitado, prevaleciendo líneas de carácter horizontal, como las del Banco de la Nación Argentina y el subsidio a las tasas de interés en los créditos a las PyMEs por parte de la Subsecretaría de la Pequeña y Mediana Empresa. Las líneas de orientación sectorial son aún casos aislados aunque en aumento, apareciendo recientemente una línea del BNA y otra del BICE para la adquisición de maquinaria agrícola nacional, un programa del BICE para la financiación de inversiones en los sectores productores de bienes de capital, y los créditos para sectores de alta tecnología en el marco del Ministerio de CyT (como los del FONSOFT).

\section{La política hacia los bienes de capital}

Un sector clave por sus características y que merece un capítulo aparte es el complejo de bienes de capital. Por las externalidades que genera al difundir progreso técnico hacia el resto del tejido productivo y por los eslabonamientos que propicia al estrechar los vínculos entre proveedores y clientes, desarrollar un sector de bienes de capital competente y dinámico debe ser un objetivo de cualquier política de desarrollo (Suzigan \& Furtado, 2006). Así lo ha entendido Brasil desde el comienzo de su estrategia industrialista en los 1950, otorgándole un carácter estratégico que lo ha llevado a convertirse en el único productor relevante de América Latina. La Argentina, en cambio, no manifiesta haber tenido al desarrollo de este sector entre sus prioridades en el período analizado - en contraste con lo que ocu-

\footnotetext{
${ }^{8}$ El BNDES-Prosoft para las empresas de software; el BNDES-Profarma para fármacos y medicamentos; la extensión de la línea Finame-Componentes a la demanda de semi-conductores y otros componentes electrónicos; BNDES-PSI, Modermaq y las líneas de la agencia Finame para la oferta y demanda de bienes de capital exclusivamente nacionales, además de otra línea especial para plantas "llave en mano". También cuentan con programas de estas características el sector aeronáutico y el automotriz.
} 
rría hacia fines de los 1950s y 1960s-, aunque recientemente ha habido algunas señales en tal sentido.

Durante los 1990, la penetración de insumos y equipos importados en el proceso productivo creció fuertemente, tanto en Brasil como en la Argentina, como consecuencia de la apertura y de la apreciación cambiaria. Fue así como se produjo cierta desarticulación en las cadenas productivas y una pérdida del valor agregado en el sector -particularmente en algunas ramas como los bienes de capital mecánicos y los componentes electrónicos.

Sin embargo, en Brasil, diversas medidas gubernamentales permitieron que algunos segmentos tuvieran un muy buen desempeño durante la década, lo que atenuó las consecuencias negativas de la apertura sobre el sector en general. En algunos casos, el trato privilegiado a los proveedores nacionales surgió directamente de las leyes de concesión, mientras que en otros se lo impuso como condicionamiento para acceder a los beneficios fiscales y facilidades financieras (particularmente del Sistema BNDES). Esto se observó principalmente en el sector de telecomunicaciones, de petróleo y en el Régimen Automotriz -que exigía un índice de nacionalización mínimo del $60 \%$ en los insumos utilizados por las terminales. $\mathrm{Al}$ mismo tiempo la industria aeronáutica mostró una fuerte expansión asociada a las ganancias de productividad posteriores a la privatización de Embraer y al fuerte apoyo financiero del BNDES, que ya hemos señalado.

Esta estrategia marca un claro contraste con lo sucedido en la Argentina, donde las privatizaciones significaron el fin del "compre nacional”. En un lapso mínimo el poder de compra estatal había desaparecido y sus herederos en el control de los servicios públicos no estaban obligados a reemplazarlo (Schvarzer, 1998). Ello afectó en forma adversa a la industria doméstica y, particularmente, a la de bienes de capital.

El régimen establecido para los bienes de capital en la Argentina consistió en la exención arancelaria para los importados, compensada con un bono fiscal a los fabricantes nacionales de BK por un valor equivalente al 14\% de su valor agregado (que era el nivel tarifario modal hasta el momento) para ser aplicado al pago de impuestos nacionales. Esta política cumplió con su objetivo de abaratar y promover las inversiones pero fue muy deficiente en la promoción de la oferta interna del sector (Sirlin, 1997).

Brasil, a diferencia de la Argentina, optó por mantener una tarifa del 14\% para la importación de BK. Y sus incentivos a la adquisición de bienes de capital han discriminado siempre en favor de la industria local, aún cuando ello implicara en ciertas ocasiones el encarecimiento de la inversión o una restricción a las políticas de promoción de las inversiones. En este sentido, todas las medidas que involucran la concesión de reducciones arancelarias para BK importados (ya sean nuevos o usados) se limitan exclusivamente a aquellos capítulos en los que no exista un similar nacional. Estas distintas estrategias implementadas por Argentina y Brasil dentro del trade-off entre promoción horizontal de la inversión y protección sectorial estratégica generan un impacto económico algo distinto, más proteccionista en el caso brasileño. 
Y por el lado financiero, asimismo, el BNDES sólo financia importaciones de bienes de capital que no cuenten con un similar nacional, mientras que la Agencia Especial de Financiamiento Industrial (FINAME) se limita a financiar exclusivamente la adquisición de maquinaria de fabricación brasileña. De esta manera, busca promover la modernización y el aumento de la eficiencia de la estructura productiva y al mismo tiempo favorecer la producción local de bienes de capital. Las líneas de financiamiento del BNA y del BICE, en cambio, sólo discriminan a favor de la producción nacional en algunos segmentos específicos, como en la maquinaria agrícola.

En síntesis, la Argentina ha tenido como objetivo fundamental el crecimiento de la inversión agregada de la economía, relegando la protección de la industria local de bienes de capital a un segundo plano a través de medidas compensatorias de carácter defensivo. Mientras tanto, Brasil priorizó la protección de su industria de Bienes de capital aún a costa de encarecer la inversión en maquinaria importada, contrarrestando parcialmente tal efecto vía ex-tarifario. Las asimetrías en cuanto al peso de dicho sector en la industria de cada país permiten explicar en parte esta percepción tan distinta de los costos-beneficios de su protección.

\section{La política de innovaciones}

La cuestión de la innovación ha merecido una especial atención en las políticas públicas brasileñas. Muestra una continuidad que se remonta a los planes industriales de los años 1960 y 1970s. Esto se ha manifestado en altos niveles de gasto en I\&D como porcentaje del PBI en relación al resto de América Latina. La Argentina, en cambio, ha transitado por mayores inconstancias y altibajos en la materia.

Durante la segunda mitad de los años 90 el Estado brasilero se preocupó por minimizar los efectos negativos de la presión de la competencia externa sobre la dotación de recursos asignados a actividades de I\&D. Por un lado, fueron creados los Fondos Sectoriales, con el objetivo de generar una fuente estable de financiamiento del gasto en I\&D para las empresas públicas y privadas. Estos fondos, financiados con impuestos sobre la facturación líquida de las empresas de cada sector, regalías etc., permitieron mantener (y hasta incrementar) los recursos para la I\&D durante el proceso de reformas (Furtado, 2008).

Por otro lado, se establecieron ratios mínimos de gasto en I\&D sobre la facturación para que las empresas pudieran acceder a ciertos beneficios fiscales y financieros o para permitir el ingreso del capital extranjero hacia algunos sectores estratégicos, buscando evitar que las filiales brasileñas de las multinacionales delegaran dichas tareas a sus casas matrices. Tal es el caso de los sectores de electrónica y la informática, donde se impusieron condicionamientos de gasto mínimo en I\&D. En las empresas en que el Estado conservó el control mayoritario, como Petrobras, se mantuvieron en funcionamiento los laboratorios de I\&D y se crearon fondos específicos para incrementar su caudal de recursos. En los sectores que se 
privatizaron, el Gobierno apuntó a preservar las estructuras de I\&D (Furtado, 2008). En el caso de Telebras, el departamento de I\&D fue separado de la empresa y convertido en fundación antes de su privatización. A los efectos de su financiación se creó el Fondo para el Desarrollo Tecnológico de las Telecomunicaciones (FUNTTEL).

En la Argentina, en cambio, durante los años 1990 sólo se vieron avances aislados en esta dirección, como la creación del Fondo Tecnológico Argentino (FONTAR) en 1996. Fue así como estas actividades se mantuvieron muy limitadas y concentradas sobre las instituciones públicas y las universidades, que además sufrieron recortes presupuestarios.

En cuanto a las empresas multinacionales, tanto por los condicionamientos impuestos en Brasil al ingreso de IED como por su propia estrategia regional, éstas tendieron a tomar a este país como headquarters dentro del mercado del Cono Sur. En materia de I\&D, las filiales brasileñas asumieron ciertas responsabilidades de alcance regional en las áreas de diseño e innovación tras la formación del Mercosur, mientras que en la Argentina incluso se recortaron actividades previamente realizadas en dichas áreas (López, 2008).

Durante los años 2000, los Gobiernos de Brasil y (más recientemente) de Argentina han dado un nuevo impulso a las políticas de innovación, incrementando el caudal de recursos públicos asignados y actualizando el marco legal para la promoción de actividades de I\&D.

En el caso de Brasil específicamente, las normas legales le permiten al Estado participar minoritariamente y en forma temporal en el capital de empresas privadas con perfil innovador, y autoriza su participación en Sociedades de Capital de Riesgo. Esta modalidad del Estado como socio en la empresa innovadora es una característica particular de Brasil que no se observa en otros países de la región (Jiménez, 2007).

En este marco, Brasil incrementó la asignación de recursos públicos a I\&D (a un $0,59 \%$ del PBI en 2007,), mientras que el nuevo régimen de incentivos fiscales y de subvenciones económicas permitió al BNDES ampliar su espacio para financiar actividades de I\&D y proyectos innovadores. Los Fondos Sectoriales tomaron una relevancia creciente en materia de incentivos a la innovación a partir de 2005, aumentando las inversiones en ciencia y tecnología de manera exponencial.

En la Argentina, se observa un cambio favorable durante los últimos años, a partir del aumento de los fondos destinados a las Instituciones de Ciencia y Técnica y de la creación, en 2007, del Ministerio de Ciencia, Tecnología e Innovación Productiva como organismo coordinador de esta área.

Sin embargo, aún se observan asimetrías muy abultadas en ambos países en relación al volumen de recursos destinados a $I \& D$, y particularmente a los gastos realizados por el sector privado. Brasil es el único país latinoamericano que destina 
más del $1 \%$ de su PBI a actividades de I\&D, y sólo su porción privada es superior a la totalidad del gasto argentino. ${ }^{9}$

Como señala Katz (2008), las firmas argentinas continúan prestando poca atención a la realización de esfuerzos locales en I\&D de nuevas tecnologías y capacidades de organización de la producción y basan su estrategia productiva en la importación de bienes de capital del exterior, en la toma de licencias internacionales de producto y en la producción de bienes altamente estandarizados, con escaso grado de diferenciación.

\section{REFLEXIONES FINALES}

Durante el presente trabajo, hemos observado que el crecimiento de la industria brasileña y de sus exportaciones ha presentado un patrón tecnológicamente más complejo en relación al argentino durante todo el período analizado, reforzando las asimetrías en el contenido tecnológico de sus estructuras productivas. Esta situación puede verse modificada a partir del proceso de revaluación que la moneda brasileña ha sufrido en los últimos tiempos pero los efectos de las políticas de largo plazo persisten sobre ambas economías.

Es que a partir del análisis comparativo de las políticas públicas implementadas a lo largo del período, podemos sostener que algunas de ellas podrían haber contribuido al fenómeno señalado. Nos referimos a aquéllas que se relacionan con cuestiones clave en materia de desarrollo y en las que Brasil ha logrado mantener en el tiempo políticas de Estado, en contraste con los vaivenes de las políticas económicas de la Argentina.

La consecución de planes industriales y de competitividad, la selección coherente y constante de los sectores estratégicos y, en particular, el apoyo al sector productor de bienes de capital, la disponibilidad de instrumentos para el financiamiento de proyectos productivos estratégicos, el fomento a la innovación y el rol asignado al capital extranjero en la economía, son temas en los que se observan asimetrías considerables entre ambos países y que seguramente hayan repercutido en sus capacidades y patrones de producción y exportaciones, impactando, en efecto, sobre el comercio bilateral.

Sin desestimar algunos cambios de rumbo favorables que se han empezado a observar durante los últimos años, el principal desafío con el que se enfrenta hoy la Argentina es en gran medida similar al de hace dos décadas: "se trata de conformar una estructura productiva que mejore la inserción comercial de nuestro país, incremente la generación de empleo productivo, reduzca la heterogeneidad estructural y, de este modo, mejore la distribución del ingreso y reduzca la pobreza extrema” (Rosales, 1988).

\footnotetext{
${ }^{9}$ Brasil destina el $1,1 \%$ del PBI a actividades de I\&D (el sector privado participa con el 0.52 ) contra el $0.51 \%$ que destina la Argentina (sólo el 0.16 corresponde al sector privado).
} 


\section{REFERENCIAS BIBLIOGRÁFICAS}

ADIMRA (2010). Asimetrías fiscales y financieras entre Argentina y Brasil. Análisis comparativo del sector de bienes de capital.

Agencia Nacional de Desarrollo de Inversiones, República Argentina. Incentivos a la Inversión. Ventajas de invertir hoy. Enero 2009.

Baruj, Gustavo; Kosacoff, Bernardo; y Porta, Fernando (2005). Políticas nacionales y la profundización del Mercosur. El impacto de las políticas de competitividad. Banco Interamericano de Desarrollo. DEPARTAMENTO DE INTEGRACIÓN Y PROGRAMAS REGIONALES. Río de Janeiro, 6-7 de julio.

Bekerman, Marta y Dalmasso, Gonzalo (2010). Políticas económicas y asimetrías productivas en Argentina y Brasil. ¿Cómo estamos hoy? CENES, Documento de Trabajo $\mathrm{N}^{\circ} 20$, Universidad de Buenos Aires.

Bekerman, Marta y Montagu, Haroldo (2007). Transformaciones recientes en el comercio entre Argentina y Brasil: impacto de los factores macro y microeconómicos. CENES, Documento de Trabajo $\mathrm{N}^{\circ}$ 17, Universidad de Buenos Aires.

Bekerman, Marta; Sirlin, Pablo; y Streb, María Luisa (1995). Las nuevas orientaciones de política industrial y de promoción de exportaciones en Argentina y Brasil. Asimetrías y posibilidades de coordinación. CENES, Documento de Trabajo N ${ }^{\circ}$ 1, Universidad de Buenos Aires.

Bernat, Gonzalo y Corso, Eduardo (2010). Dualidad innovativa en la Argentina: Fundamentos macro y microeconómicos. Boletín Informativo Techint $\mathrm{N}^{\circ} 331$.

Bezchinsky, G.; Dinenzon, M.; Guissani, L.; Caino, O.; López, B.; y Amiel, S. (2007) Inversión Extranjera Directa en la Argentina. Crisis, reestructuración y nuevas tendencias después de la Convertibilidad. En Kosacoff, Bernardo (ed.) (2007). Crisis, recuperación y nuevos dilemas. La economía argentina 2002-2007. Oficina de la Cepal en Buenos Aires, Santiago de Chile, noviembre. Capítulo V, p. 149-186.

Bouzas, Roberto y Cabello, Sebastián M. (2007). La formulación de la política comercial en la Argentina: fundamentos estructurales e institucionales de la volatilidad. En Marcos Sawaya Jank y Simão Davi Silber (coordinadores). Politicas comerciales comparadas: modelos organizacionales y su desempeño. Editora Singular, São Paulo.

Casa Civil da Presidência da República, Brasil (noviembre de 2003). Diretrizes de Política Industrial, Tecnológica e de Comércio Exterior.

Fanelli, J. M. y Frenkel, R. (1994) Estabilidad y estructura: interacciones en el crecimiento económico. CEDES, Buenos Aires, Argentina.

Fanelli J.M (2008). Mercosur, integracióny profundización de los mercados financieros. En Fanelli, José María; Albrieu, Ramiro; Beckzuk, Ricardo; Pires de Souza, Francisco; Cardim de Carvalho, Fernando; Rutkoski, Cecilia; Aboal, Diego; Lanzilotta, Bibliana; y Perera, Marcelo (2008). Mercosur: integración y profundización de los mercados financieros. Serie Red Mercosur $\mathrm{N}^{\circ} 9$.

Ferraz, João Carlos; Pires de Souza Francisco; y Kupfer David (2010) Trayectorias para el desarrollo brasileño. Boletín Informativo Techint No 333.

Furtado, André. (2008) O sistema brasileiro da inovação e o Mercosul - Dinâmica interativa, políticas públicas e limitações estruturais. En Rozenwurcel, G.; Gianella, C.; López, Andrés. (2008). El sistema nacional de innovación en la Argentina y los desafíos de la integración regional. En Rozenwurcel, G.; Gianella, C.; Bezchinsky, G. y Thomas, H. Innovación a escala Mercosur. Una vía para superar el estancamiento regional. Buenos Aires.

Jiménez, Luis Felipe (2007). Capital de riesgo y mecanismos financieros de apoyo a la innovación en Brasil y Chile. Cepal, $\mathrm{N}^{\circ} 177$, Santiago de Chile.

Katz, Jorge (2008). La economía argentina en el tercer milenio: ciencia, tecnología y desarrollo. Boletín informativo de Techint $\mathrm{N}^{\circ} 327$, Buenos Aires.

López, Andrés. (2008). El sistema nacional de innovación en la Argentina y los desafíos de la integración regional. En Rozenwurcel, G.; Gianella, C.; Bezchinsky, G. y Thomas, H. Innovación a escala Mercosur. Una vía para superar el estancamiento regional. Buenos Aires. Capítulo III, p. 81.

Penido de Freitas, María Cristina y Magalhaes Prates, Daniela (2000). La experiencia de apertura financiera en Argentina, Brasil y México. Revista de la Cepal, abril 2000; p. 53-69. 
Peres, Wilson y Primi, Annalisa (2009). Theory and Practice of Industrial Policy. Evidence from de Latin American Experience. Cepal, Santiago de Chile, febrero.

Schvarzer, Jorge (1998). La industria argentina en la tormenta de los 90. Revista Nueva sociedad Nro. 158; p. 139-159.

Sirlin, Pablo (1997). Evaluación de la política de bienes de capital en la Argentina, Revista de la Cepal, $\mathrm{N}^{\circ}$ 61. Santiago de Chile, Cepal.

Suzigan, Wilson y Furtado, João (2006). Política industrial y desarrollo. Revista de la Cepal $\mathrm{N}^{\mathrm{o}} 89$, Santiago de Chile. 
ANEXO

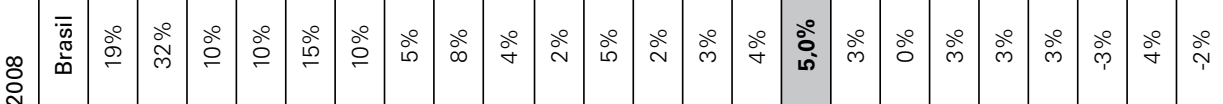

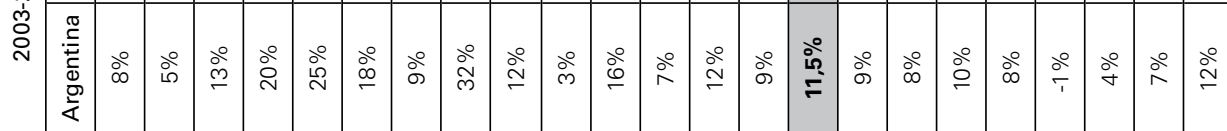

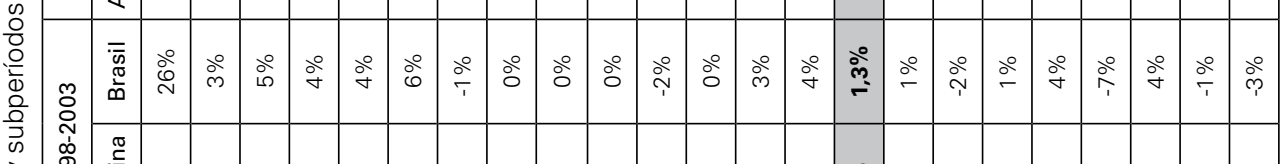

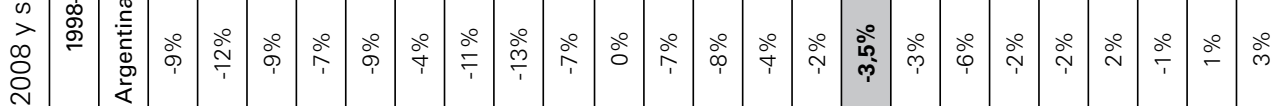

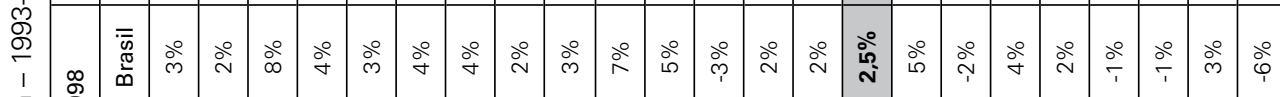

:흥

它 兽

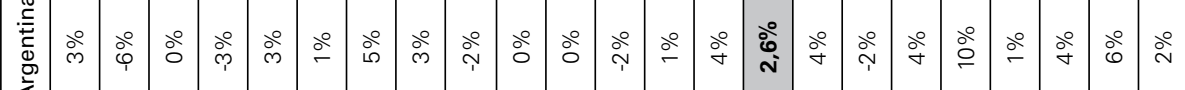

$\frac{10}{0}$

$\stackrel{\frac{8}{6}}{\frac{.0}{4}}$

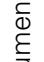

$\frac{3}{9}$

ब

莗

$\cdot \frac{\Phi}{\bar{\varepsilon}}$

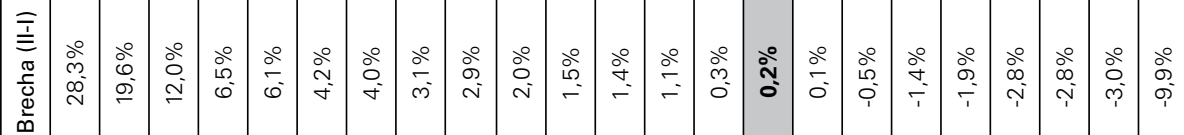

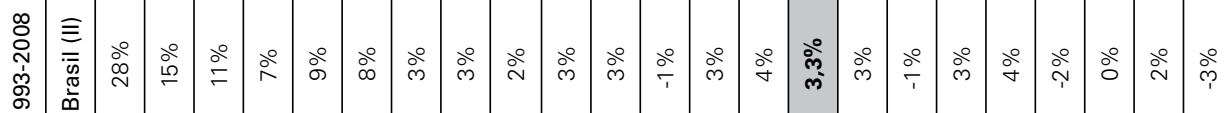

$\equiv$

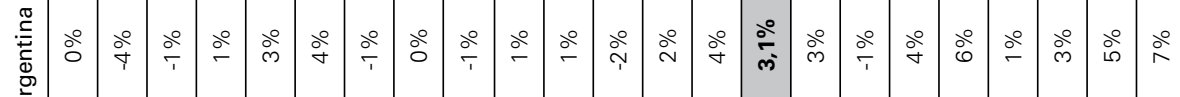

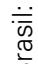

竞

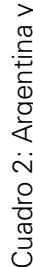

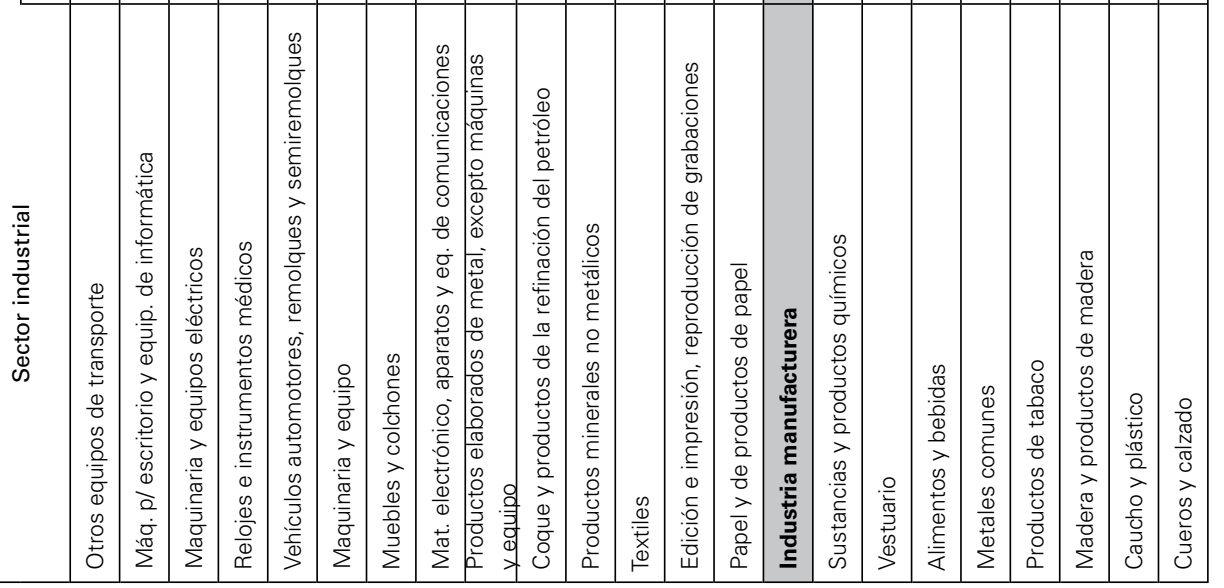

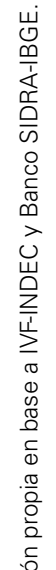




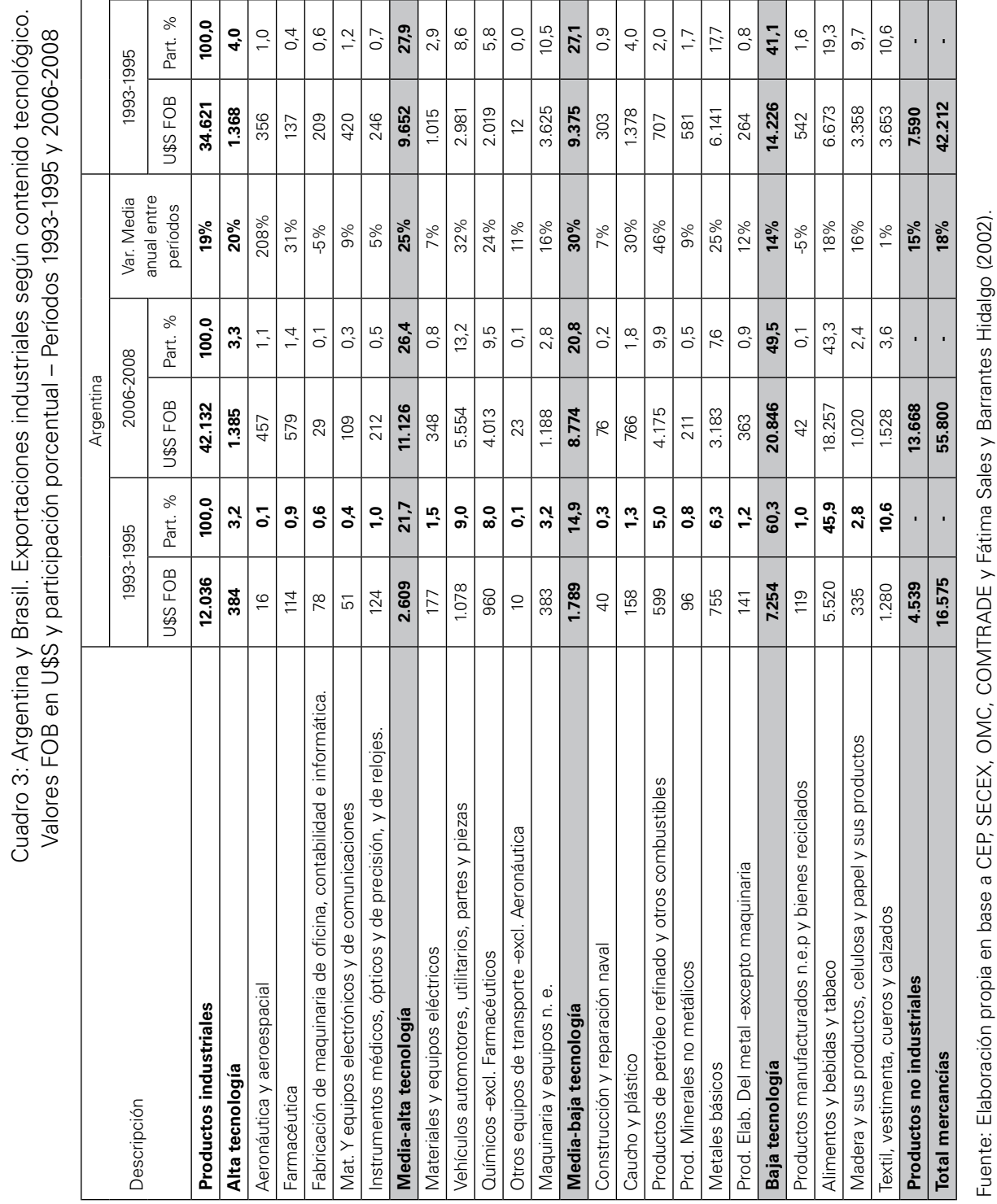

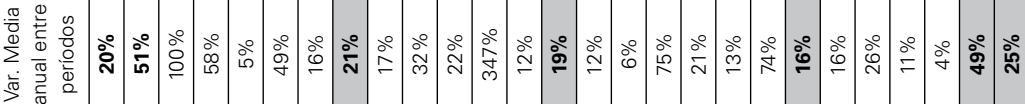

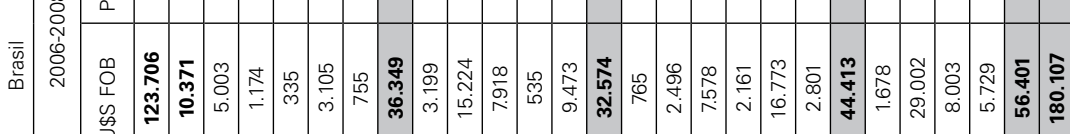

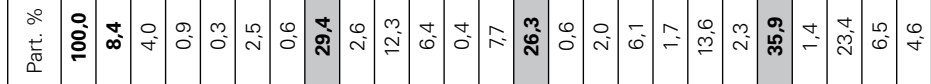

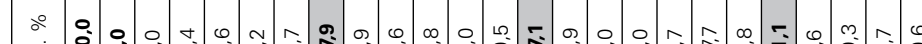


Alain Herscovici, Aldo Musacchio, Alkimar Moura, Ana Maria Bianchi, Ana Maria Malik, André Cunha, André Marques, André Singer, Anita Kon, Antonio Correa de Lacerda, Carmem Feijó, Claudia Helena Cavalieri, Claudia Heller, Claudio Couto, Claudio Dedecca, Claudio Vilar Furtado, Clesio Xavier, Ernesto Lozardo, Evaldo Alves, Fabio Gallo, Fabrício Pereira da Silva, Fernando Barbi, Fernando Cardim de Carvalho, Flavio Riani, Flavio Vilela Vieira, Floriano Peixoto de Azevedo Marques, Francisco Lopes, Gabriel Caldas Montes, George Avelino, Jair Antunes, João Henrique Pederiva, João Mergulhão, João Saboia, João Sicsú, José Carlos Braga, José Eduardo Faria, José Luis Oreiro, Julia de Medeiros Braga, Lauro Mattei, Lenina Pomeranz, Leonardo Weller, Ligia Vasconcellos, Luiz Antonio de Oliveira Lima, Luiz Fernando de Paula, Manoel Carlos de Castro Pires, Marcelo Nonnenberg, Marcos Antonio Macedo Cintra, Marco Flavio da Cunha Resende, Marcos Tostes Lamonica, Maria Cristina Cacciamali, Maria de Lourdes Mollo, Maria Rita Loureiro, Mario Possas, Mauricio Coutinho, Newton Paulo Bueno, Paulo Gala, Paulo Picchetti, Pedro Cesar Dutra Fonseca, Pedro Drago, Pedro Rossi, Pierre-Richard Ágenor, Renato Baumann, Rodolfo Hoffmann, Rogério P. de Andrade, Rosa Maria Vieira, Rubens Barbosa, Rubens Penha Cysne, Sergio de Azevedo, Sérgio Magno Mendes, Sergio Sauer, Simon Schwartzman, Sonia Draibe, Tania de Toledo Lima, Thompson de Almeida Andrade, Vera Thorstensen e Victor Pelaez 